\title{
LOOKING FOR COMPACTNESS IN SOBOLEV SPACES ON NONCOMPACT METRIC SPACES
}

\author{
Przemysław Górka \\ Warsaw University of Technology, Department of Mathematics and Information Sciences \\ Ul. Koszykowa 75, 00-662 Warsaw, Poland; pgorka@mini.pw.edu.pl
}

\begin{abstract}
We study Sobolev spaces on noncompact metric measure spaces. We show compact embedding of $H$-invariant Sobolev spaces, where $H$ is a subgroup of all isometries preserving the measure on the metric measure space.
\end{abstract}

\section{Introduction}

Let $\Omega$ be an open subset of the Euclidean space $\mathbf{R}^{n}$. If the boundary of $\Omega$ is sufficiently regular and $p<n$, then the Sobolev embedding $W^{1, p}(\Omega) \hookrightarrow L^{q}(\Omega)$ holds, where $p \leq q \leq p^{*}:=\frac{n p}{n-p}$. Additionally, if the set $\Omega$ is bounded, then the RellichKondrachov compactness theorem $W^{1, p}(\Omega) \hookrightarrow \hookrightarrow L^{q}(\Omega)$ is satisfied, where $p \leq q<p^{*}$ (see e.g. [1]). Furthermore, if $\Omega$ is not bounded, then compactness not necessarily holds. Indeed, by taking $\Omega=\mathbf{R}$, nonzero $\phi \in C_{0}^{\infty}(\mathbf{R})$ such that $\operatorname{supp} \phi \subset\left[-\frac{1}{2}, \frac{1}{2}\right]$ and $u_{n}(x)=\phi(x-n)$, we obtain that $u_{n}$ is bounded in $W^{1, p}(\mathbf{R})$, but there does not exist a subsequence of $u_{n}$ that converges in $L^{q}(\mathbf{R})$.

On the other hand, suppose that $n \geq 2$ and define the subspace of $W^{1, p}\left(\mathbf{R}^{n}\right)$ consisting of radially symmetric functions (invariant under the action of ortogonal group $O(n))$, i.e.,

$$
W_{r}^{1, p}\left(\mathbf{R}^{n}\right):=\left\{u \in W^{1, p}(\mathbf{R})^{n} \mid u \text { is radially symmetric }\right\} .
$$

Then it was shown by Berestycki-Lions [4, 33], (see also Coleman-Glazer-Martin [9] and Strauss [37]) that

$$
W_{r}^{1, p}\left(\mathbf{R}^{n}\right) \hookrightarrow \hookrightarrow L^{q}\left(\mathbf{R}^{n}\right)
$$

where $p<q<p^{*}$. The Berestycki-Lions type theorem has also been established on Riemannian manifolds (see Hebey-Vaugon [27] and Skrzypczak-Tintarev [35]). Namely, assume that $G$ is a compact subgroup of the group of global isometries of the complete Riemannian manifold $(M, g)$. Then, under some additional assumptions on the geometry of $(M, g)$ and some assumptions on the orbits under the action of $G$, the following compact embedding holds $W_{G}^{1, p}(M, g) \hookrightarrow \hookrightarrow L^{q}(M, g)$, where $W_{G}^{1, p}(M, g)$ is the subspace of $W^{1, p}(M, g)$ consisting of $G$-invariant functions. Moreover, Balogh and Kristály proved the Berestycki-Lions compactness on the Heisenberg group [3]. More recently, the compact embedding results of the Berestycki-Lions type have been extended to the generalized Lebesgue-Sobolev spaces $W^{1, p(\cdot)}$ (see Fan-ZhaoZhao in the Euclidean case [11] and Gaczkowski-Górka-Pons in the case of complete Riemannian manifolds [13]).

The main objective of the paper is to prove the Berestycki-Lions compactness type theorem (see Theorem 3.2) on metric measure spaces. Roughly speaking, we

https://doi.org/10.5186/aasfm.2018.4332

2010 Mathematics Subject Classification: Primary 46E35, 30L99.

Key words: Sobolev spaces, metric measure spaces, compact embedding, Berestycki-Lions theorem, covering lemma, isometries preserving measure. 
aim to prove the following result. Suppose that $(X, \rho, \mu)$ is a metric measure space equipped with $s$-Ahlfors regular measure and $H$ is a subgroup of the measurepreserving isometries of $(X, \rho)$. Then, by employing some assumption on the orbits under the action of $H$, we have that the following compact embedding holds $M_{H}^{1, p}(X) \hookrightarrow \hookrightarrow L^{q}(X)$, where $p<q<p^{*}:=\frac{s p}{s-p}$ and $M_{H}^{1, p}(X)$ is $H$-invariant HajłaszSobolev space.

Finally, we collect known results about compactness theorems for $M^{1, p}$ spaces. Kałamajska has proved compactness of the embedding $M^{1, p}$ in $L^{p}$ assuming that the measure is finite (see Theorem 2 in [31]). Tilli and Ambrosio showed that the embedding $M^{1, p} \hookrightarrow \hookrightarrow L^{q}$, where $q<p^{*}=\frac{s p}{s-p}$ holds for compact metric measure spaces with lower $s$-Ahlfors measure (see Theorem 5.4 .3 in [2] ). Recently, compactness results have been proved for variable exponent Hajłasz-Sobolev spaces $M^{1, p(\cdot)}(X)$, where $X$ is a compact metric space (see $[12,19]$ ).

The remainder of the paper is structured as follows. In Section 2, we introduce the notations and recall the notion of Sobolev spaces on general metric measure spaces. We also formulate and prove the covering lemma there. Our principal assertion, concerning the compact embedding of the Hajłasz-Sobolev spaces on noncompact metric spaces, is formulated and proven in Section 3. Some open problems are contained in the final part.

\section{Preliminaries}

Let $(X, \rho, \mu)$ be a metric measure space equipped with a metric $\rho$ and the Borel regular measure $\mu$. We assume throughout the paper that the measure of every open nonempty set is positive and that the measure of every bounded set is finite. Additionally, we assume that the measure $\mu$ satisfies a doubling condition. It means that, there exists a constant $C_{d}>0$ such that for every ball $B(x, r)$,

$$
\mu(B(x, 2 r)) \leq C_{d} \mu(B(x, r)) .
$$

It is well known (see e.g. Lemma 14.6 in [23]) that the doubling condition implies that, there exists a positive constant $D$ satisfying

$$
D\left(\frac{r_{1}}{r_{2}}\right)^{s} \leq \frac{\mu\left(B\left(x_{1}, r_{1}\right)\right)}{\mu\left(B\left(x_{2}, r_{2}\right)\right)}, \quad \text { where } s=\log _{2} C_{d},
$$

for all balls $B\left(x_{2}, r_{2}\right)$ and $B\left(x_{1}, r_{1}\right)$ with $r_{2} \geq r_{1}>0$ and $x_{1} \in B\left(x_{2}, r_{2}\right)$. It follows from the above inequality that if $X$ is bounded, then there exists $b>0$ such that the following inequality holds for $r<\operatorname{diam} X$

$$
\mu(B(x, r)) \geq \frac{1}{b} r^{s} .
$$

On the other hand, if the metric measure space equipped with a doubling measure is not bounded, then inequality (1) does not necessarily hold.

In majority part of our paper we shall assume that the metric measure space $(X, \rho, \mu)$ is Ahlfors $s$-regular (we also say that $(X, \rho, \mu)$ is a metric measure space with $s$-regular measure $\mu$ ). It means that there exists a constant $b$ such that

$$
\frac{1}{b} r^{s} \leq \mu(B(x, r)) \leq b r^{s}
$$

for all balls $B(x, r) \subset X$ with $r<\operatorname{diam} X$.

We are now in a position to recall the notion of Sobolev spaces on metric measure spaces [21]. Let $(X, \rho, \mu)$ be a metric measure space. We say that a $p$-integrable 
function $f$ belongs to the Hajłasz-Sobolev space $M^{1, p}(X)$ if there exists $g \in L^{p}(X)$, called a generalized gradient, such that

$$
|f(x)-f(y)| \leq \rho(x, y)(g(x)+g(y)) \quad \text { a.e. for } \quad x, y \in X .
$$

We equip the space $M^{1, p}(X)$ with the norm

$$
\|f\|_{M^{1, p}(X)}=\|f\|_{L^{p}(X)}+\inf \|g\|_{L^{p}(X)},
$$

where the infimum is taken over all the generalized gradients. Then $M^{1, p}$ is a Banach space. For the basic properties of this kind of spaces, we refer to $[2,21,22,23,29$, $28,32]$.

Suppose that $f$ is locally integrable and $A$ is a measurable set then by $f_{A}$ we denote the integral average of the function $f$ over the set $A$, i.e.,

$$
f_{A}:=f_{A} f d \mu=\frac{1}{\mu(A)} \int_{A} f d \mu .
$$

To prove the main theorems we shall need the following result.

Proposition 2.1. [15] Let $(X, \rho, \mu)$ be a metric measure space with s-regular measure $\mu$. If $s>p$, then

$$
M^{1, p}(X) \hookrightarrow L^{p^{*}}(X),
$$

where $p^{*}=\frac{s p}{s-p}$. Moreover, there exists $C=C(s, p, b)$, depending on $s, p, b$, such that for each $u \in M^{1, p}(X)$, the following inequality holds

$$
\|u\|_{L^{p^{*}}(X)} \leq C\left(\|u\|_{L^{p}(X)}+\|g\|_{L^{p}(X)}\right) .
$$

Furthermore, if diam $X=\infty$, then

$$
\|u\|_{L^{p^{*}(X)}} \leq C\|g\|_{L^{p}(X)} .
$$

We need also the notion of isometries preserving the measure. Let $\operatorname{Iso}(X)$ be the group of isometries of the metric space $(X, \rho)$, then the group of measure-preserving isometries of $(X, \rho, \mu)$ consists of all isometries of $X$ preserving the measure $\mu$ :

$$
\operatorname{Iso}_{\mu}(X)=\left\{\phi \in \operatorname{Iso}(X) \mid \phi_{\#} \mu=\mu\right\},
$$

where $\phi_{\#} \mu$ is the pushforward measure. It is also noteworthy that in the case of Riemannian manifolds, we have that $\operatorname{Iso}_{V_{g}}(M, g)=\operatorname{Iso}(M, g)$, where by $V_{g}$ we denoted the Riemannian measure. For a deeper discussion of measure-preserving isometries we refer the reader to [36]. In further considerations $H$ denotes a fixed subgroup of $\operatorname{Iso}_{\mu}(X)$. As usual, the orbit of $x \in X$ under the action of $H$ is the set $H(x)=$ $\{h(x) \mid h \in H\}$. Given $x$ in $X$ and $R>0$, we consider the following quantity

$$
M_{H}(x, R)=\sup \left\{\operatorname{Card}\left\{x_{i}\right\}_{i \in I} \mid x_{i} \in H(x), B\left(x_{i}, R\right) \cap B\left(x_{j}, R\right)=\emptyset \text { for } i \neq j\right\} .
$$

$M_{H}(x, R)$ gives the lowest upper bound for the number of non overlapping $R$-balls in the orbit of $x$.

In addition, we denote by $M_{H}^{1, p}(X)$ the subspace of $M^{1, p}(X)$ consisting of $H$ invariant functions. Since $H$ is a subgroup of measure-preserving isometries, one can easily convince oneself that $M_{H}^{1, p}(X)$ is a closed subspace of $M^{1, p}(X)$. Therefore, $M_{H}^{1, p}(X)$ is a Banach space.

2.1. Covering lemma. In this subsection we shall prove the following covering lemma.

Lemma 2.1. Let $(X, \rho, \mu)$ be a metric measure space with doubling measure and $r>0$. Then, there exists a sequence $\left\{x_{i}\right\} \subset X$ such that for any $\delta>r$ : 
a) $X=\bigcup_{i} B\left(x_{i}, r\right)$;

b) For any $x \in X, x$ belongs to at most $C_{d}^{6}\left(\frac{\delta}{r}\right)^{\log _{2} C_{d}}$ balls $B\left(x_{i}, \delta\right)$.

Proof. Since the measure is doubling, the metric space $X$ is separable. Let $A$ be a countable and dense subset of $X$. Put

$$
X(r)=\left\{\left\{x_{i}\right\} \subset A \mid \forall_{i \neq j}, \rho\left(x_{i}, x_{j}\right) \geq \frac{r}{2}\right\} .
$$

By the Zorn-Kuratowski Lemma, there exists a maximal element $\left\{x_{i}\right\}$ in $X(r)$. Thus, since $A$ is dense and $\left\{x_{i}\right\}$ is a maximal element, we get

$$
X=\bigcup_{i \in J} B\left(x_{i}, r\right) \text {. }
$$

This completes the proof of a).

Thus, it remains to prove b). For this purpose, we fix $x \in X$ and $\delta>r$. Set

$$
J_{\delta}(x)=\left\{i \in J \mid x \in B\left(x_{i}, \delta\right)\right\} .
$$

It is easily seen that for $i \in J_{\delta}(x)$, we have

$$
B\left(x_{i}, \frac{r}{4}\right) \subset B(x, 2 \delta) \subset B\left(x_{i}, 4 \delta\right) .
$$

Since $B\left(x_{i}, \frac{r}{4}\right) \cap B\left(x_{j}, \frac{r}{4}\right)=\emptyset$ for $i \neq j$, then by the elementary properties of measures and by doubling condition, we obtain

$$
\begin{aligned}
\mu(B(x, 2 \delta)) & \geq \mu\left(\bigcup_{i \in J_{\delta}(x)} B\left(x_{i}, \frac{r}{4}\right)\right)=\sum_{i \in J_{\delta}(x)} \mu\left(B\left(x_{i}, \frac{r}{4}\right)\right) \\
& \geq C_{d}^{-2}\left(\frac{r}{16 \delta}\right)^{\log _{2} C_{d}} \sum_{i \in J_{\delta}(x)} \mu\left(B\left(x_{i}, 4 \delta\right)\right) \\
& \geq C_{d}^{-6}\left(\frac{r}{\delta}\right)^{\log _{2} C_{d}} \sum_{i \in J_{\delta}(x)} \mu(B(x, 2 \delta)) \\
& =C_{d}^{-6}\left(\frac{r}{\delta}\right)^{\log _{2} C_{d}} \operatorname{Card}\left(J_{\delta}(x)\right) \mu(B(x, 2 \delta)) .
\end{aligned}
$$

Consequently, we get

$$
\operatorname{Card}\left(J_{\delta}(x)\right) \leq C_{d}^{6}\left(\frac{\delta}{r}\right)^{\log _{2} C_{d}},
$$

which completes the proof of the covering lemma.

\section{Compactness}

In this section of our paper we state and prove our principal assertion. We will start with the proof of the following result.

Theorem 3.1. Suppose that $(X, \rho, \mu)$ is a metric measure space with s-regular measure $\mu$. Assume in addition that $u_{n}$ is a bounded sequence in $M^{1, p}(X)$, where $p<s$ and, there exists $r>0$ such that

$$
\lim _{n \rightarrow \infty} \sup _{y \in X} \int_{B(y, r)}\left|u_{n}\right|^{p} d \mu=0 .
$$

Then,

$$
u_{n} \rightarrow 0 \quad \text { in } L^{q}(X)
$$


where $p<q<p^{*}=\frac{s p}{s-p}$.

Proof. The strategy of the proof is similar to the proof of Lemma 1.21 from [38]. Let us fix $x_{0} \in X$ and define the following Lipschitz map

$$
f_{x_{0}, r}(x):= \begin{cases}\frac{1}{r}\left(2 r-\rho\left(x, x_{0}\right)\right) & \text { if } x \in B\left(x_{0}, 2 r\right) \backslash\left(x_{0}, r\right), \\ 1 & \text { if } x \in B\left(x_{0}, r\right), \\ 0 & \text { if } x \in X \backslash B\left(x_{0}, 2 r\right),\end{cases}
$$

where the Lipschitz constant $L_{r}=\frac{1}{r}$ does not depend on $x_{0}$.

We will show that if $u \in M^{1, p}(\stackrel{r}{X})$ with the generalized gradient $g$, then $f_{x_{0}, r} u \in$ $M^{1, p}(X)$. Indeed, taking

$$
\tilde{g}(x)=\left(|u(x)| L_{r}+g(x)\right) \chi_{B\left(x_{0}, 2 r\right)},
$$

one can easily prove that $\tilde{g}$ is the Hajłasz gradient of $f_{x_{0}, r} u$.

Now, let us recall the following interpolation inequality. Suppose that $\nu \leq \sigma \leq \alpha$ and $u \in L^{\nu} \cap L^{\alpha}$. Then, $u \in L^{\sigma}$ and

$$
\|u\|_{L^{\sigma}} \leq\|u\|_{L^{\nu}}^{\theta}\|u\|_{L^{\alpha}}^{1-\theta}
$$

where

$$
\frac{1}{\sigma}=\frac{\theta}{\nu}+\frac{1-\theta}{\alpha}
$$

We are now in a position to prove our theorem. We may assume that $\left\|u_{n}\right\|_{M^{1, p}(X)}<$ 1. Let $\epsilon \leq \frac{(q-p)^{2}}{2 p}$, such that

$$
p<q<t:=q+\epsilon<p^{*} .
$$

It is easy to see that for such $\epsilon$, we have

$$
\frac{q-p}{q-p+\epsilon} \frac{q+\epsilon}{p} \geq 1
$$

Furthermore, by the interpolation inequality and Proposition 2.1, we have

$$
\left\|u_{n} f_{x_{0}, r}\right\|_{L^{t}(X)} \leq C(t, p, s, b)\left\|u_{n} f_{x_{0}, r}\right\|_{M^{1, p}(X)} .
$$

Thus, taking into account the above considerations, we get

$$
\begin{aligned}
\left\|u_{n}\right\|_{L^{t}\left(B\left(x_{0}, r\right)\right)} & \leq C(t, p, s, b)\left(\left\|u_{n}\right\|_{L^{p}\left(B\left(x_{0}, 2 r\right)\right)}+\left\|\tilde{g_{n}}\right\|_{L^{p}\left(B\left(x_{0}, 2 r\right)\right)}\right) \\
& \leq C(t, p, s, b, r)\left(\left\|u_{n}\right\|_{L^{p}\left(B\left(x_{0}, 2 r\right)\right)}+\left\|g_{n}\right\|_{L^{p}\left(B\left(x_{0}, 2 r\right)\right)}\right) .
\end{aligned}
$$

Additionally, using the interpolation inequality with $\sigma=q, \nu=p, \alpha=t$, we obtain

$$
\begin{aligned}
\int_{B(y, r)}\left|u_{n}\right|^{q} d \mu & \leq\left(\int_{B(y, r)}\left|u_{n}\right|^{p} d \mu\right)^{\frac{t-q}{t-p}}\left(\int_{B(y, r)}\left|u_{n}\right|^{t} d \mu\right)^{\frac{q-p}{t-p}} \\
& =\left(\int_{B(y, r)}\left|u_{n}\right|^{p} d \mu\right)^{\frac{\epsilon}{q-p+\epsilon}}\left(\int_{B(y, r)}\left|u_{n}\right|^{q+\epsilon} d \mu\right)^{\frac{q-p}{q-p+\epsilon}} .
\end{aligned}
$$

Subsequently, denoting $\tau_{n}=\sup _{y \in X}\left(\int_{B(y, r)}\left|u_{n}\right|^{p} d \mu\right)^{\frac{\epsilon}{q-p+\epsilon}}$ and using inequality (2), we get

$$
\begin{aligned}
& \int_{B(y, r)}\left|u_{n}\right|^{q} d \mu \leq \tau_{n} C(t, p, s, b, r)^{\frac{t-q}{t-p} \alpha}\left(\left\|u_{n}\right\|_{L^{p}\left(B\left(x_{0}, 2 r\right)\right.}+\left\|g_{n}\right\|_{L^{p}\left(B\left(x_{0}, 2 r\right)\right.}\right)^{\frac{q-p}{q-p+\epsilon}(q+\epsilon)} \\
& \leq \tau_{n} D(t, p, q, r, s, b)\left(\left(\int_{B\left(x_{0}, 2 r\right)}\left|u_{n}\right|^{p} d \mu\right)^{\frac{q-p}{q-p+\epsilon} \frac{q+\epsilon}{p}}+\left(\int_{B\left(x_{0}, 2 r\right)}\left|g_{n}\right|^{p} d \mu\right)^{\frac{q-p}{q-p+\epsilon} \frac{q+\epsilon}{p}}\right) .
\end{aligned}
$$


Since $\left\|u_{n}\right\|_{M^{1, p}(X)}<1$ and $\frac{q-p}{q-p+\epsilon} \frac{q+\epsilon}{p} \geq 1$, we may write that

$$
\int_{B(y, r)}\left|u_{n}\right|^{q} d \mu \leq \tau_{n} D(t, p, q, r, s, b)\left(\int_{B(y, 2 r)}\left|u_{n}\right|^{p} d \mu+\int_{B(y, 2 r)}\left|g_{n}\right|^{p} d \mu\right) .
$$

Thus, using the covering lemma, we have

$$
\begin{aligned}
\int_{X}\left|u_{n}\right|^{q} d \mu & \leq \sum_{i=1}^{\infty} \int_{B\left(x_{i}, r\right)}\left|u_{n}\right|^{q} d \mu \leq \tau_{n} D(t, p, q, r, s, b) \sum_{i=1}^{\infty} \int_{B\left(x_{i}, 2 r\right)}\left(\left|u_{n}\right|^{p}+\left|g_{n}\right|^{p}\right) d \mu \\
& \leq \tau_{n} D(t, p, q, r, s, b) C_{d}^{7} \int_{X}\left(\left|u_{n}\right|^{p}+\left|g_{n}\right|^{p}\right) d \mu \leq \tau_{n} D(t, p, q, r, s, b) C_{d}^{7},
\end{aligned}
$$

where $C_{d}=b^{2} 2^{s}$ is the doubling constant for $s$-regular measure. Finally, we conclude

$$
\lim _{n \rightarrow \infty} \int_{X}\left|u_{n}\right|^{q} d \mu=0,
$$

which completes the proof of Theorem 3.1.

Our next main result is the following claim.

Theorem 3.2. Assume that $(X, \rho, \mu)$ is a metric measure space with s-regular measure $\mu$ such that $M^{1, p}(X)$ is reflexive. Moreover, let $H \triangleleft \operatorname{Iso}_{\mu}(X)$ be such that

$$
\lim _{R \rightarrow \infty} \inf _{x \in X \backslash B\left(x_{0}, R\right)} M_{H}(x, r)=\infty,
$$

where $x_{0}$ is any fixed point of $X$ and $r>0$. If $s>p>1$, then for any $q$ such that $p<q<p^{*}$, we have the compact embedding

$$
M_{H}^{1, p}(X) \hookrightarrow \hookrightarrow L^{q}(X) .
$$

Remark. If the metric measure space equipped with a doubling measure supports a Poincaré inequality, then the space $M^{1, p}(X)$ is reflexive. Indeed, from Theorem 4.9 in [34] we know that if $X$ is a metric measure space equipped with a doubling measure and $X$ supports $q$-Poincaré inequality for some $q<p$, then $M^{1, p}(X)=N^{1, p}(X)$, where $N^{1, p}(X)$ is a Newton-Sobolev space. On the other hand, by the Cheeger reflexivity theorem (see [7] and for an elementary proof we refer the reader to [10]), we have that $N^{1, p}(X)$ is reflexive, provided the measure $\mu$ on $X$ is doubling and $X$ supports a $p$-Poincaré inequality.

Example 1. Let $(M, g)$ be a complete Riemannian manifold. Assume that the Ricci curvature Ric is nonnegative, then by the Bishop-Gromov comparison theorem (see for instance [8]) we have that the metric measure space $\left(M, V_{g}, d_{g}\right)$ is doubling, where $V_{g}$ is the Riemannian measure and $d_{g}$ stands for the geodesic distance. Furthermore, by the Buser isoperimetric inequality [5] we get that $\left(M, V_{g}, d_{g}\right)$ supports a Poincaré inequality.

Example 2. Let $\left(\mathbf{H}_{1}, d, l_{3}\right)$ be the first Heisenberg group equipped with a Carnot metrid $d$ and the Lebesgue measure $l_{3}$ (see [6] and the references given there). Then, the measure $l_{3}$ is doubling, in fact the space is 4 -regular. Moreover, $\left(\mathbf{H}_{1}, d, l_{3}\right)$ supports a 1-Poincaré inequality [30].

Proof. By Proposition 2.1, we have $M_{H}^{1, p}(X) \hookrightarrow L^{q}(X)$. For the rest of the proof the following lemma is needed.

Lemma 3.1. For $x_{0} \in X$ and $R>0$, we define the operator

$$
F_{x_{0}, R}: M^{1, p}(X) \rightarrow L^{p}(X)
$$


as follows

$$
F_{x_{0}, R}(u)=u f_{x_{0}, R}
$$

If the assumptions of Theorem 3.2 are satisfied, then $F_{x_{0}, R}$ is compact.

Proof. Let $u_{n}$ be a bounded sequence in $M^{1, p}(X)$. We need to show that the sequence $F_{x_{0}, R}\left(u_{n}\right)$ is relatively compact in $L^{p}(X)$. For this purpose, we shall use the characterization of relatively compact sets in $L^{p}(X)$ from [18]. For convenience of the reader, we briefly recall this result. Namely, the subset $\mathcal{F}$ of $L^{p}(X)$ is relatively compact in $L^{p}(X)$ if and only if $\mathcal{F}$ is bounded, has uniform $L^{p}$-decay and is uniformly $L^{p}$-equicontinous.

Since in our case, the proofs of boundedness and uniform $L^{p}$-decay are straightforward, we show that the family $u_{n} f_{x_{0}, R}$ is uniformly $L^{p}$-equicontinous. Let $r>0$. We have

$$
\begin{aligned}
& \int_{X}\left|u_{n} f_{x_{0}, R}(x)-\left(u_{n} f_{x_{0}, R}\right)_{B(x, r)}\right|^{p} d \mu(x) \\
& \leq \int_{X}\left|\frac{1}{\mu(B(x, r))} \int_{B(x, r)}\right| u_{n} f_{x_{0}, R}(x)-u_{n} f_{x_{0}, R}(y)|d \mu(y)|^{p} d \mu(x) \\
& \leq \int_{X}\left|\frac{r}{\mu(B(x, r))} \int_{B(x, r)}\right| \tilde{g}_{n}(x)+\tilde{g}_{n}(y)|d \mu(y)|^{p} d \mu(x) \\
& \leq \int_{X}\left|r\left(\left|\tilde{g}_{n}(x)\right|+\frac{1}{\mu(B(x, r))} \int_{B(x, r)}\left|\tilde{g}_{n}(y)\right| d \mu(y)\right)\right|^{p} d \mu(x) \\
& \leq 2^{p-1} r^{p}\left(\int_{X}\left|\tilde{g}_{n}(x)\right|^{p} d \mu(x)+\int_{X} \mid \frac{1}{\mu(B(x, r))} \underset{B(x, r)}{\int \tilde{g}_{n}(y)|d \mu(y)|^{p} d \mu(x)}\right) \\
& \leq 2^{p-1} r^{p}\left(\int_{X}\left|\tilde{g}_{n}(x)\right|^{p} d \mu(x)+\int_{X}\left|\mathcal{M}\left(\tilde{g}_{n}\right)(x)\right|^{p} d \mu(x)\right)
\end{aligned}
$$

where $\mathcal{M}$ is a maximal function.

By virtue of the Hardy-Littlewood maximal function theorem $\|\mathcal{M}(v)\|_{L^{p}(X)} \leq$ $C_{p}\|v\|_{L^{p}(X)}$ (see e.g. [28]), we get

$$
\begin{aligned}
\int_{X}\left|u_{n} f_{x_{0}, R}(x)-\left(u_{n} f_{x_{0}, R}\right)_{B(x, r)}\right|^{p} d \mu(x) & \leq 2^{p-1} r^{p}\left(1+C_{p}^{p}\right) \int_{X}\left|\tilde{g}_{n}(x)\right|^{p} d \mu(x) \\
& \leq 2^{2 p-2} r^{p}\left(1+C_{p}^{p}\right)\left(1+L_{R}^{p}\right)\left\|u_{n}\right\|_{M^{1, p}(X)} .
\end{aligned}
$$

Therefore, the proof of Lemma 3.1 directly follows from Theorem 1 in [18].

We are now in a position to proceed further with the proof of Theorem 3.2. We take a bounded sequence $u_{n} \in M_{H}^{1, p}(X)$. In view of reflexivity, we may assume that, there exists a subsequence, still denoted by $\left\{u_{n}\right\}$, that weakly converges to some $u$ in $M_{H}^{1, p}(X)$. Thus, the sequence $v_{n}:=u_{n}-u$ converges weakly to 0 in $M_{H}^{1, p}(X)$. 
Since the measure $\mu$ is $H$-invariant, for each $y_{1} \in X$ and for every $y_{2} \in H\left(y_{1}\right)$ we have

$$
\int_{B\left(y_{1}, r\right)}\left|v_{n}(x)\right|^{p} d \mu=\int_{B\left(y_{2}, r\right)}\left|v_{n}(x)\right|^{p} d \mu .
$$

Therefore, for every $y$ in $X$,

$$
M(y, r) \int_{B(y, r)}\left|v_{n}(x)\right|^{p} d \mu \leq \int_{X}\left|v_{n}(x)\right|^{p} d \mu \leq C .
$$

Relation (3) and the assumptions on $M(y, r)$ ensure that for each $\epsilon>0$, there exists some finite $R_{\epsilon}$ such that for every $n$

$$
\sup _{y \in X \backslash B\left(x_{0}, R_{\epsilon}\right)} \int_{B(y, r)}\left|v_{n}(x)\right|^{p} d \mu \leq \frac{C}{\inf _{y \in X \backslash B\left(x_{0}, R_{\epsilon}\right)} M(y, r)} \leq \epsilon .
$$

Hence, by Lemma 3.1 and the Cantor diagonal method, we may construct a subsequence $v_{n}$ such that

$$
\lim _{n \rightarrow \infty} \sup _{y \in X} \int_{B(y, r)}\left|v_{n}(x)\right|^{p} d \mu=0 .
$$

Finally, this and Theorem 3.1 imply desired result stated in Theorem 3.2.

\section{Final remarks}

We summarize the paper with open problems.

Problem 1. Let $M^{1, p(\cdot)}(X, \rho, \mu)$ be the Hajłasz-Sobolev space with the variable exponent $p(\cdot)$ (see $[12,24,25,26]$ for definition). Does Theorem 3.2 hold under some condition on the exponent $p$ ?

Problem 2. Let $G$ be a locally compact abelian group and $G^{\wedge}$ denote the dual group. Then, for $s>0$ and map $\gamma: G^{\wedge} \rightarrow[0, \infty)$ we define Sobolev space $H_{\gamma}^{s}(G)$ (see $[20,17,16])$ as follows. We shall say that $f \in L^{2}(G)$ belongs to the Sobolev space $H_{\gamma}^{s}(G)$ if

$$
\int_{G^{\wedge}}\left(1+\gamma(\xi)^{2}\right)^{s}|\hat{f}(\xi)|^{2} d \hat{\mu}_{G}(\xi)<\infty
$$

Moreover, for $f \in H_{\gamma}^{s}(G)$ its norm $\|f\|_{H_{\gamma}^{s}(G)}$ is defined as follows

$$
\|f\|_{H_{\gamma}^{s}(G)}=\left(\int_{G^{\wedge}}\left(1+\gamma(\xi)^{2}\right)^{s}|\hat{f}(\xi)|^{2} d \hat{\mu}_{G}(\xi)\right)^{\frac{1}{2}} .
$$

Suppose that $H$ is a group acting on $G$ and $H_{\gamma, H}^{s}(G)$ stands for the subspace of $H_{\gamma}^{s}(G)$ consisting of $H$-invariant functions. The problem is: find conditions implying compact embedding of $H_{\gamma, H}^{s}(G)$.

Acknowledgements. Some part of the paper has been performed during the visit of the author to the TIFR Centre for Applicable Mathematics in Bangalore. P.G. wish to thank for its hospitality. The author thanks Marcin Dudziński for reading the preliminary version of this manuscript. Moreover, the author would also like to thank the anonymous referees for providing several comments and suggestions. 


\section{References}

[1] Adams, R. A.: Sobolev spaces. - Pure and Applied Mathematics 65, Academic Press, New York-London, 1975.

[2] Ambrosio, L., and P. Tilli: Topics on analysis in metric spaces. - Oxford Lecture Ser. Math. Appl. 25, Oxford Univ. Press, Oxford, 2004.

[3] BAlogh, Z. M., and A. KRIStÁly: Lions-type compactness and Rubik actions on the Heisenberg group. - Calc. Var. Partial Differential Equations 48, 2013, 89-109.

[4] Berestycki, H., and P.L. Lions: Existence of a ground state in nonlinear equations of the Klein-Gordon type. Variational inequalities and complementarity problems. - In: Proc. Internat. School, Erice, 1978, Wiley, Chichester, 1980, 35-51.

[5] Buser, P.: A note on the isoperimetric constant. - Ann. Sci. Éc. Norm. Supér. (4) 15, 1982, 213-230.

[6] Capogna, L., D. Danielli, S. D. Pauls, and J. T. Tyson: An introduction to the Heisenberg group and the sub-Riemannian isoperimetric problem. - Progr. Math. 259, Birkhäuser Verlag, Basel, 2007.

[7] Cheeger, J.: Differentiability of Lipschitz functions on metric measure spaces. - Geom. Funct. Anal. 9, 1999, 428-517.

[8] Cheeger, J., M. Gromov, and M. Taylor: Finite propagation speed, kernel estimates for functions of the Laplace operator and the geometry of complete Riemannian manifolds. - J. Differential Geom. 17, 1982, 15-53.

[9] Coleman, S., V. Glazer, and A. Martin: Action minima among solutions to a class of Euclidean scalar field equations. - Comm. Math. Phys. 58, 1978, 211-221.

[10] Durand-Cartagena, E., and N. Shanmugalingam: An elementary proof of Cheeger's theorem on reflexivity of Newton-Sobolev spaces of functions in metric measure spaces. - J. Anal. 21, 2013, 73-83.

[11] Fan, X., Y. Zhao, and D. ZhaO: Compact imbedding theorems with symmetry of StraussLions type for the space $W^{1, p(x)}(\Omega)$. - J. Math. Anal. Appl. 255:1, 2001, 333-348.

[12] Gaczkowski, M., and P. GórkA: Variable Hajłasz-Sobolev spaces on compact metric spaces. - Math. Slov. 67, 2017, 199-208.

[13] Gaczkowski, M., P. Górka, and D. J. Pons: Sobolev spaces with variable exponents on complete manifolds. - J. Funct. Anal. 270, 2016, 1379-1415.

[14] Gaczkowski, M., P. Górka, and D. J. Pons: Corrigendum to "Sobolev spaces with variable exponents on complete manifolds". - J. Funct. Anal. 272, 2017, 1296-1299.

[15] Górka, P.: In metric-measure spaces Sobolev embedding is equivalent to a lower bound for the measure. - Potential Anal. 47, 2017, 13-19.

[16] Górka, P., and T. Kostrzewa: Sobolev spaces on metrizable groups. - Ann. Acad. Sci. Fenn. Math. 40, 2015, 837-849.

[17] Górka, P., T. Kostrzewa, and E. G. Reyes: Sobolev spaces on locally compact abelian groups: compact embeddings and local spaces. - J. Funct. Spaces 2014, 2014, ID 404738.

[18] Górka, P., and A. Macios: Riesz-Kolmogorov theorem on metric spaces. - Miskolc Math. Notes 15, 2014, 459-465.

[19] Górka, P., and A. Macios: Almost everything you need to know about relatively compact sets in variable Lebesgue spaces. - J. Funct. Anal. 269:7, 2015, 1925-1949.

[20] Górka, P., and E. G. Reyes: Sobolev spaces on locally compact abelian groups and the bosonic string equation. - J. Aust. Math. Soc. 98, 2015, 39-53.

[21] HajŁasz, P.: Sobolev spaces on an arbitrary metric space. - Potential Anal. 5, 1996, 403-415.

[22] HajŁasz, P.: Sobolev spaces on metric-measure spaces. - In: Heat kernels and analysis on manifolds, graphs, and metric spaces (Paris, 2002), Contemp. Math. 338, 2003, 173-218. 
[23] HajŁasz, P., and P. Koskela: Sobolev met Poincaré. - Mem. Amer. Math. Soc. 688, 2000, $1-101$.

[24] Harjulehto, P., P. Hästö, and V. Latvala: Sobolev embeddings in metric measure spaces with variable dimension. - Math. Z. 254, 2006, 591-609.

[25] Harjulehto, P., P. Hästö, and M. Pere: Variable exponent Lebesgue spaces on metric spaces: The Hardy-Littlewood maximal operator. - Real Anal. Exchange 30, 2004, 87-104.

[26] Harjulehto, P., P. HÄstö, and M. Pere: Variable exponent Sobolev spaces on metric measure spaces. - Funct. Approx. Comment. Math. 36, 2006, 79-94.

[27] Hebey, E., and M. VAugon: Sobolev spaces in the presence of symmetries. - J. Math. Pures Appl. 76, 1997, 859-881.

[28] Heinonen, J.: Lectures on analysis on metric spaces. - Universitext, 2001.

[29] Heinonen, J., P. Koskela, N. Shanmugalingam, and J. T. Tyson: Sobolev spaces on metric measure spaces. An approach based on upper gradients. - New Math. Monogr. 27, Cambridge Univ. Press, Cambridge, 2015.

[30] Jerison, D.: The Poincaré inequality for vector fields satisfying Hörmander's condition. Duke Math. J. 53, 1986, 503-523.

[31] KaŁamajska, A.: On compactness of embedding for Sobolev spaces defined on metric spaces. - Ann. Acad. Sci. Fenn. Math. 24, 1999, 123-132.

[32] Kinnunen, J., and O. Martio: The Sobolev capacity on metric spaces. - Ann. Acad. Sci. Fenn. Math. 21, 1996, 367-382.

[33] Lions, P. L.: Symétrie et compacité dans les espaces de Sobolev. - J. Funct. Anal. 49, 1982, $315-334$.

[34] Shanmugalingam, N.: Newtonian spaces: an extension of Sobolev spaces to metric measure spaces. - Rev. Mat. Iberoam. 16, 2000, 243-279.

[35] Skrzypczak, L., and C. Tintarev: A geometric criterion for compactness of invariant subspaces. - Arch. Math. 101, 2013, 259-268.

[36] SosA, G.: The isometry group of an $\mathrm{RCD}^{\star}$-space is Lie. - arXiv:1609.02098.

[37] Strauss, W. A.: Existence of solitary waves in higher dimensions. - Comm. Math. Phys. 55, 1977, 149-162.

[38] Willem, M.: Minimax theorems. - Progr. Nonlinear Differential Equations Appl. 24, Birkhäuser Boston, Inc., Boston, MA, 1996.

Received 29 May 2017 • Accepted 6 October 2017 